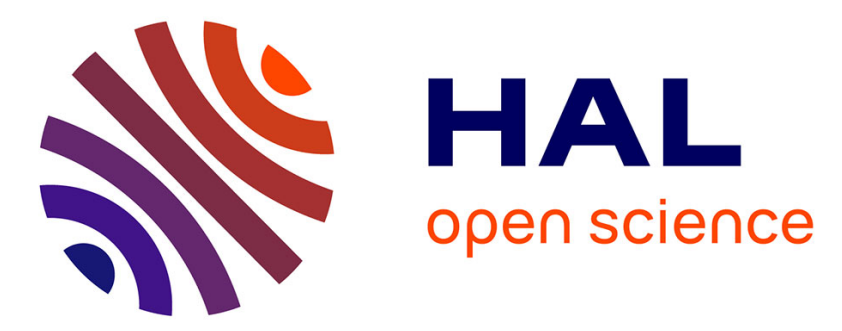

\title{
Attosecond imaging of molecular electronic wavepackets
}

Stefan Haessler, J Caillat, W Boutu, C Giovanetti-Teixeira, T Ruchon, T Auguste, Z Diveki, P Breger, A Maquet, B Carré, et al.

\section{To cite this version:}

Stefan Haessler, J Caillat, W Boutu, C Giovanetti-Teixeira, T Ruchon, et al.. Attosecond imaging of molecular electronic wavepackets. Nature Physics, 2010, 6, pp.200. 10.1038/NPHYS1511 . hal01164779

HAL Id: hal-01164779

https://hal-ensta-paris.archives-ouvertes.fr/hal-01164779

Submitted on 17 Jun 2015

HAL is a multi-disciplinary open access archive for the deposit and dissemination of scientific research documents, whether they are published or not. The documents may come from teaching and research institutions in France or abroad, or from public or private research centers.
L'archive ouverte pluridisciplinaire HAL, est destinée au dépôt et à la diffusion de documents scientifiques de niveau recherche, publiés ou non, émanant des établissements d'enseignement et de recherche français ou étrangers, des laboratoires publics ou privés. 


\title{
Attosecond imaging of molecular electronic wave-packets
}

S. Haessler ${ }^{1}$, J. Caillat ${ }^{2,3}$, W. Boutu ${ }^{1}$, C. Giovanetti-Teixeira ${ }^{2,3}$, T. Ruchon ${ }^{1}$, T. Auguste ${ }^{1}$, Z. Diveki $^{1}$,

\author{
P. Breger ${ }^{1}$, A. Maquet ${ }^{2,3}$, B. Carré ${ }^{1}$, R. Taïeb ${ }^{2,3}$, P. Salières $^{1}$
}

1 CEA-Saclay, IRAMIS, Service des Photons, Atomes et Molécules, 91191 Gif-sur-Yvette, France.

2 UPMC Univ. Paris 06, UMR 7614, Laboratoire de Chimie Physique-Matière et Rayonnement, 11 rue Pierre et Marie Curie, 75231 Paris Cedex 05, France.

3 CNRS, UMR 7614, LCPMR F-75005 Paris, France.

A strong laser field may tunnel ionize a molecule from several orbitals simultaneously, forming an attosecond electron-hole wave-packet. Both temporal and spatial information on this wavepacket can be obtained through the coherent soft $\mathrm{X}$-ray emission resulting from the laserdriven recollision of the liberated electron with the core. By characterizing the emission from aligned $\mathrm{N}_{2}$ molecules, we evidence the attosecond contributions of the two highest occupied molecular orbitals. We determine conditions where they are disentangled in the real and imaginary parts of the emission dipole moment. This allows us to perform a tomographic reconstruction of both orbitals with Ångström spatial resolution. Their coherent superposition provides experimental images of the attosecond wavepacket created in the ionization process. Our results open the perspective of imaging ultrafast intra-molecular dynamics combining attosecond and Ångström resolutions.

The recent development of attosecond sources of coherent soft X-rays has opened the perspective of observing and controlling the fastest electronic processes in matter. In this rapidly evolving field, the attosecond processes explored up to now may be grouped in two categories. The first one encompasses the dynamics driven by a strong laser field in atoms and molecules. The laser period being a few femtoseconds, the timescale of sub-cycle electron dynamics is attosecond, as in tunnelling ionization $^{1,2}$ and continuum dynamics ${ }^{3-7}$. The second category deals with 'intrinsic' (laser-free) 
dynamics that is characteristic of the system: conduction band dynamics ${ }^{8}$ or bound-state dynamics related to ultrashort lived transient states ${ }^{9-11}$. Attosecond 'intrinsic' dynamics could also be driven by coherent superposition of bound states. Such coherent superposition of bound states determines, e.g., intra-molecular electron transfer via non-adiabatic transitions in chemical reactions ${ }^{12}$. Theoretical studies investigated for instance electron-electron correlation ${ }^{13,14}$ or charge migration in small peptides ${ }^{15}$, and only recently both theoretical and experimental work has been performed in the context of tunnel ionization ${ }^{16,17}$.

Imaging such coherent wavepackets localized in time and space would allow a better understanding of their dynamics. High-order harmonic generation (HHG) can be an efficient tool for such purpose. HHG occurs when a strong laser pulse, of frequency $\omega_{\mathrm{L}}$, tunnel-ionizes a gas target, creating an electron wave-packet (EWP) that is subsequently accelerated and driven back to the core where it recombines, emitting an attosecond burst of coherent soft X-rays ${ }^{18-20}$. Advanced characterization of the latter gives extremely precise information on both the structure and the dynamics of the radiating system. As the EWP associated with the observed photon emission has a duration at recollision of a few hundred attoseconds, it allows probing ultrafast dynamics in the excited molecular system (ion and correlated continuum electron) such as ultrafast nuclear motion ${ }^{21,22}$, or attosecond electron dynamics in the recombination process ${ }^{23}$. As the EWP de Broglie wavelength $\lambda_{\mathrm{dB}}$ is of the order of molecular internuclear distances $(\sim 1 \AA)$, it allows observing quantum interferences in the recombination process ${ }^{23-27}$. It may also lead to a tomographic reconstruction of the radiating orbital with $\AA$ resolution, as first proposed by Itatani et $\mathrm{al}^{28}$. This fascinating possibility of spatially imaging the wave function of a quantum object in amplitude and phase attracted much attention. However both the assumptions made in the theoretical treatment and the incomplete experimental data raised intense discussions ${ }^{29-34}$ on the feasibility of the technique that is still to be demonstrated.

Here we study an attosecond wavepacket created in aligned nitrogen molecules by tunnel ionization from both the highest occupied molecular orbital (HOMO), and the lower-lying orbital (referred to as HOMO-1). The HHG emission resulting from the freed EWP recollision is characterized in amplitude and phase. In our generation conditions, the signatures of the two orbitals can be disentangled, allowing their tomographic reconstruction. Exploiting further the measured relative phase of their 
contributions allows us to reconstruct the dynamic superposition of the orbitals left empty after tunnel ionization (so called "hole"), which evolves on the attosecond time-scale before recombination. This hole can be seen as a "negative" image of the ion left in a coherent superposition of the ground (X) and excited (A) states.

\section{Creating and probing a bound attosecond wavepacket}

Recent experimental and theoretical studies ${ }^{16,17,35}$ have revealed that molecules interacting with a strong laser field could be tunnel ionized from several valence orbitals simultaneously due to their small energy separations and different geometries. This was observed in the resulting HHG emission, where the different orbitals lead to interfering contributions in the $q$ th harmonic total dipole moment $\vec{D}\left(\theta, q \omega_{\mathrm{L}}\right)$, where $\theta$ is the angle between the laser polarization and the molecular axis. In the strong field approximation (SFA), when only the НОМО, $\psi^{\text {номо }}$, and НОМО-1, $\psi^{\text {номо-1 }}$, contribute without coupling, $\vec{D}\left(\theta, q \omega_{\mathrm{L}}\right) \operatorname{reads}^{28,36}$ :

$$
\begin{aligned}
\vec{D}\left(\theta, q \omega_{\mathrm{L}}\right) & =a_{1}\left(\theta, k_{q}\right) \int\left[\psi^{\text {номо }}(\vec{r})\right]^{*} \vec{r} \mathrm{e}^{\mathrm{i} \vec{k}_{q} \cdot \vec{r}} \mathrm{~d} \vec{r}+a_{2}\left(\theta, k_{q}\right) \int\left[\psi^{\text {номо- }}(\vec{r})\right]^{*} \vec{r} \mathrm{e}^{\mathrm{i} \vec{k}_{q} \cdot \vec{r}} \mathrm{~d} \vec{r} \\
& \equiv a_{1}\left(\theta, k_{q}\right) \vec{d}^{\text {номо }}\left(\theta, k_{q}\right)+a_{2}\left(\theta, k_{q}\right) \vec{d}^{\text {номо- }}\left(\theta, k_{q}\right)
\end{aligned}
$$

where the bound wave functions are expressed in the Koopmans approximation ${ }^{37}$ and the scattering state in the plane-wave approximation, with $q \omega_{\mathrm{L}}=k_{q}{ }^{2} / 2$ (discussed later, atomic units are used throughout). The complex wave packet amplitude $a_{1 / 2}$ of each contribution results from the tunnelionization and continuum excursion steps. The angle $\theta$ determines the ratio of the orbital contributions in both the ionization amplitude and the recombination dipole moment ${ }^{16,17}$. The HHG emission thus contains rich structural and dynamical information on the complex ionic system but this information is not accessible through measurement of the harmonic intensity only. Our experimental setup achieves laser-driven non-adiabatic molecular alignment, subsequent attosecond pulse generation and characterization in the spectral domain in amplitude and phase of the HHG polarization component parallel to the driving laser (see Methods). The temporal intensity profile of the attosecond emission from $\mathrm{N}_{2}$ molecules is shown in Fig. 1a for different $\theta$. The pulse 
shape hardly varies but the peak timing presents a gradual shift by -50 as when $\theta$ increases from $0^{\circ}$ to $90^{\circ}$.

Understanding the origin of this temporal shift and its relation to multi-orbital contributions requires a detailed analysis of the effective recombination dipole $d^{\exp }(\theta, q)$ extracted from the measured spectra. To this end, one has to remove the complex amplitude accumulated by the EWP prior to recombination, which induces a chirp in the attosecond emission ${ }^{5}$, i.e., $d^{\exp }(\theta, q)=D^{\exp }(\theta, q) / a_{1}$. This is achieved by normalizing the measured XUV spectrum (amplitude and phase) for nitrogen by the spectrum for argon (same ionization potential $I_{p}$ ) obtained under the same experimental conditions, and multiplying this ratio by the theoretical recombination dipole $d_{\mathrm{Ar}}(q)$ for $\operatorname{argon}^{23,28}$. The obtained normalized amplitude (Fig. 2a) reproduces the results of ref. 28 with a spectral minimum at harmonic 25 at all alignment angles. However, the assumption made in ref. 28 of a $\pi$ phase jump associated to structural interferences (expected to be $\theta$-dependent ${ }^{24}$ ) is not confirmed here. Our measured phase difference (Fig. 2b) presents a more complex behaviour with two identified jumps. In the following, we show that this non-trivial phase is driven by not only structural interferences but also multi-orbital effects favoured by both energy and geometry criteria.

\section{Disentangling the orbital contributions to HHG}

In $\mathrm{N}_{2}$, the HOMO ( $\sigma_{\mathrm{g}}$ symmetry) and HOMO-1 ( $\pi_{\mathrm{u}}$ symmetry) are separated by only ${ }^{38} \Delta \varepsilon \approx-1.4 \mathrm{eV}$. The differences in symmetry and geometry result in a strong angular dependence of the ratio of their ionization probabilities ${ }^{39,40}$, that is maximum at $\theta=90^{\circ}$. They also lead to a larger plane-wave recombination dipole moment for the HOMO-1 at high energy, and consequently an overall significant contribution to the high-harmonic emission at $90^{\circ}$. Our analysis, focused on the dipole phase, follows the chronology of the SFA model: we address for each contribution the phase $\phi_{\mathrm{d}}=\phi_{\mathrm{c}}+\phi_{\mathrm{r}}$, with (i) the phase $\phi_{\mathrm{c}}=\arg \left(a_{1 / 2}\right)$ acquired by the ionic system during the continuum excursion and (ii) the phase $\phi_{\mathrm{r}}=\arg \left(d^{\mathrm{HOMO} / \mathrm{HOMO}-1}\right)$ added by recombination.

The dipole normalization procedure removed the phase $\phi_{\mathrm{c}}$, but only for the HOMO contribution. The HOMO- 1 contribution is left, after calibration, with a phase equal to ${ }^{41} \Delta \phi_{\mathrm{c}}\left(q \omega_{\mathrm{L}}\right)=\arg \left(a_{2} / a_{1}\right)$ 
$=\phi_{\mathrm{c}}{ }^{\text {HOMO-1 }}\left(q \omega_{\mathrm{L}}\right)-\phi_{\mathrm{c}}^{\text {HOMO }}\left(q \omega_{\mathrm{L}}\right) \approx \Delta \varepsilon \tau_{q}$ (see Supplementary Information), where $\tau_{q}$ is the electron excursion time associated with harmonic order $q$. The difference $\Delta \phi_{c}\left(q \omega_{\mathrm{L}}\right)$ is plotted in Fig.3a for our experimental conditions. It evolves slowly around $-\pi$, value reached in the middle of the experimental spectral range, at $\mathrm{H} 25$.

The recombination dipole expressed in the plane-wave (PW) approximation (appearing in Eq. (1)) turns out to be the Fourier transform of the bound wave-function times the dipole operator ${ }^{28,36}$. Due to the symmetry properties of the orbitals and of the dipole operator (antisymmetric) on the one hand, and of the Fourier transform on the other hand, the recombination dipole is purely imaginary-valued for the HOMO $\left(\sigma_{\mathrm{g}}\right)$ and purely real-valued for the HOMO-1 $\left(\pi_{\mathrm{u}}\right)$, resulting in $\Delta \phi_{\mathrm{r}}=\pi / 2$. At this stage, the accumulated phase difference $\Delta \phi_{\mathrm{d}}=\Delta \phi_{\mathrm{c}}+\Delta \phi_{\mathrm{r}}$ is close to $-\pi / 2$ : the HOMO and HOMO- 1 contributions to the normalized dipole $d^{\text {exp }}(\theta, q)$ are practically disentangled in the imaginary and real part, respectively. The measured phase variation around $\mathrm{H} 25$ at $\theta=90^{\circ}$ could be a signature of a significant contribution of HOMO-1 coming into play for the high harmonics.

However, the PW approximation assumes the recolliding electron to be free, although it is accelerated by the parent ionic core, inducing a distortion of the EWP, which may cancel out the disentanglement of the HOMO and HOMO-1 contributions. Computing exact values of molecular recombination dipoles requires a precise knowledge of the continuum states, which is by no means a simple task in such multi-electron multi-center systems ${ }^{30,42}$. Nevertheless, valuable insight can be gained through a relatively simple model, that uses Coulomb waves ${ }^{37}(\mathrm{CWs})$ for the continuum. This approach is motivated by the fact that the ionic core acts asymptotically as an effective point charge $Z^{*}=1$. Figure $3 \mathrm{~b}, \mathrm{c}$ presents the phase variation of the dipole against the electron momentum $k$ computed for the HOMO within the $\mathrm{CW}$ approximation, for three illustrative $\theta$ values. Two representative cases are considered: the plane wave limit $Z^{*}=0$ (Fig. 3b) and $Z^{*}=1$ corresponding to the effective $\mathrm{N}_{2}{ }^{+}$charge (Fig. 3c). As expected from the symmetry criteria mentioned before, in the case of PWs, the phase is an odd multiple of $\pi / 2$, the sudden jumps being a manifestation of structural sign changes in the purely imaginary dipole. For $Z^{*}=1$ and $k>0.5$ a.u, the evolution tends to follow the variations observed for $Z^{*}=0$, with the following differences: (i) besides being smoothed, the $\pi$-jumps are translated towards 
lower $k$ by an amount $\delta k \approx 0.4$ a.u. and (ii) the phases are vertically shifted by a global term which decays smoothly as $k$ increases. By assigning to each harmonic frequency $q \omega_{\mathrm{L}}$ an asymptotic momentum $k_{q}$ using $^{36} k_{q}^{2} / 2=q \omega_{\mathrm{L}}-I_{p}$, one finds that our measurements were performed in the window 0.9 a.u. $<k<1.54$ a.u. In this range, the phase variations are dominantly governed by the structural jumps also observed in the PW approximation, with a global phase shift $\delta \phi_{1} \approx \pi / 4$. We performed the same study for the HOMO-1, and obtained similar conclusions both on the jump translation and on the global phase shift. Therefore we conclude that (i) we can base our interpretation on the PW approximation, provided a proper translation is made on the electron momentum scale, and (ii) we can still consider the HOMO and HOMO-1 contributions to be disentangled in the imaginary and real parts of $d^{\exp }(\theta, q)$ respectively, provided a $-\delta \phi_{\mathrm{r}}$ rotation in the complex plane. Within the experimental $k$-range, the momentum translation in (i) corresponds to a kinetic energy translation of $\approx 15 \mathrm{eV}$. This is consistent with the heuristic relation $k_{q}{ }^{2} / 2=q \omega_{\mathrm{L}}$ generally used in the SFA interpretation of molecular harmonic spectra ${ }^{24}$. The removal of $I_{p}$ from the energy conservation law is interpreted as a compensation for the absence of electron acceleration close to the core in the PW treatment. In (ii), performing a global phase shift assumes that the exact scattering phase shift is practically independent of energy in the studied spectral range, molecular alignment, and ionized orbital - as suggested by the $\mathrm{CW}$ model. This is consistent with numerical experiments ${ }^{31}$ based on the time-dependent Schrödinger equation for a single contributing orbital, where such an overall dipole rotation allowed quasisuppression of the imaginary part of the reconstructed orbital. The physical origin of this rotation was, however, not identified in ref. 31. The global phase shift, $\delta \phi_{\mathrm{r}}$, for exact $\mathrm{N}_{2}$ scattering states is probably different from the $\mathrm{CW}$ based prediction, we thus empirically chose the phase origin that provided the most consistent reconstructions (see Fig.2 and Supplementary Information).

\section{Tomographic imaging of the dynamic hole}

Our understanding of the complex dipole structure allows us to exploit the measured data and (i) to simulate the temporal profiles of the attosecond emission, (ii) to reconstruct the involved orbitals by a 
tomographic procedure, and, merging the temporal and structural aspects, (iii) to image, at the instant of recombination, the dynamic "hole" formed by the coherent ionization channels.

Combining the predicted phases $\phi_{\mathrm{d}}$ and experimental harmonic amplitudes, we simulated the temporal intensity profile of the attosecond emission as a function of the relative weight $\left|a_{2}\right|:\left|a_{1}\right|$ of both contributions. Figure $1 \mathrm{~b}$ reproduces very well the experimental negative temporal shift of the attosecond pulse peak when a significant HOMO-1 contribution is gradually added to the HOMO contribution as the angle is increased from $0^{\circ}$ to $90^{\circ}$. This regular evolution is consistent with the phase difference close to $-\pi / 2$ that minimizes the interference of the two contributions. Different experimental conditions (laser intensity and wavelength, selection of long trajectories) can lead to stronger interferences and much more distorted temporal profiles, as illustrated by the simulations presented in Fig. $1 \mathrm{c}$ where $\Delta \phi_{1}=\pi / 2$ was omitted leading to a total phase difference centered around $-\pi$. Following the tomographic procedure suggested by Itatani et $a .^{28}$, we now define the 2D reconstructed functions:

$$
f_{\alpha}(x, y)=\frac{1}{\alpha} \sum_{q} \sum_{j} 2 \omega_{\mathrm{L}} \Delta \theta\left[d_{\alpha}^{\exp }\left(\theta_{j}, k_{q}\right)\right]^{*} \exp \left[\mathrm{i} k_{q}\left(x \cos \theta_{j}+y \sin \theta_{j}\right)\right] \quad(\alpha=x, y)
$$

where $q$ and $j$ span the harmonic and angular samplings respectively, $\Delta \theta$ is the angular step $\left(10^{\circ}\right)$, $\left[d^{\exp }{ }_{\alpha}\right]^{*}$ is the complex-conjugated $\alpha$-component of the normalized dipole and the discretized inverse Fourier transform is restricted to the $(x, y)$ plane. If the transform were performed exactly, both $f_{x}$ and $f_{y}$ would be equal to the wavefunction contributing to the dipole, integrated over the transverse direction $z$.

We present in Fig.4a,b the 2D orbitals reconstructed from our data shown in Fig.2, taken as the half sum of $f_{x}$ and $f_{y}$ in order to average the errors due to the discrete sampling. The measurements were done for angles between $0^{\circ}$ and $90^{\circ}$. The tomographic procedure requires the experimental dipole to be extrapolated up to $360^{\circ}$, by imposing the assumed symmetry associated with the orbital to be imaged. Note that new methods are currently developed to detect experimentally the orbital symmetries by controlling the EWP trajectory ${ }^{43,44}$. The disentanglement of the multi-orbital contributions in the experimental dipole allows us to reconstruct both the HOMO (from its imaginary part when selecting the $\sigma_{\mathrm{g}}$ symmetry), and the HOMO-1 (from its real part when selecting the $\pi_{\mathrm{u}}$ 
symmetry). As seen in Fig. 4a, our reconstructed $\sigma_{\mathrm{g}}$ orbital possesses the main characteristics of the nitrogen HOMO (Fig. 4e), namely three main lobes with alternating signs, separated by two nodal surfaces passing through each nucleus. The visual aspect of the HOMO-1 reconstruction (Fig. 4b) is dominated by the imposed symmetry and possesses little particular structure.

We performed simulated reconstructions for the HOMO and HOMO-1 in the PW approximation, based on orbitals calculated with GAMESS ${ }^{45}$. To mimic the experimental conditions, we computed the dipoles over the span corresponding to the harmonic and orientation samplings using the relation $k_{q}{ }^{2} / 2=q \omega_{\mathrm{L}}$, restricting the dipoles to the laser polarization orientation. The simulated HOMO reconstruction, shown in Fig. 4c, is strikingly similar to the experimental one (Fig. 4a): both present very similar distorted lobes, with extrema and nodes at the same locations. For the HOMO-1, the experimental and simulated reconstructions (Fig. 4b,d) both present a main central lobe, but with notably different sizes. We relate this to the fact that the HOMO-1 reconstructions had to be done using the velocity-form of the dipole operator, in order to avoid numerical singularities appearing when reconstructing $\pi$ orbitals in the length gauge. This was confirmed by very similar discrepancies obtained when reconstructing the HOMO in the velocity gauge (see Supplementary Information). The main distortions and extra oscillations in our reconstructions, compared to exact Hartree-Fock orbitals (shown in Fig. 4e,f), thus result essentially from the restricted harmonic span. Our analysis cannot account for the remaining deviations, in particular the HOMO:HOMO-1 contrasts (the ratio of the maximum amplitudes is 1:10 in the experiment, while 3:10 in the simulations). Our simulations do not take into account the different ionization yields of the two orbitals and possibly different continuum wavepacket spreading. Deviations may also be related to approximations inherent to the SFA, such as neglecting bound- and continuum-state distortions by the strong external field ${ }^{46,47}$. For systems with little relaxation, such as $\mathrm{N}_{2}$, the Dyson orbital (which is the orbital to be considered in a rigorous treatment) does not differ significantly from the ionized Hartree-Fock orbital and the exchange terms ${ }^{48,49}$, the magnitude of which is considerably overestimated in the plane-wave approximation, are negligible. Distortions may also result from properties of the exact dipole absent in 
our model ${ }^{30,42}$, though we expect relatively long-lived resonances to have little impact on the HHG recombination dipole due to the sub-cycle time-scale of the electron excursion.

The HOMO and HOMO-1 experimental reconstructions, $\psi_{\exp }^{\text {HОмO }}$ and $\psi_{\exp }^{\text {HOMO-1 }}$ (Fig. 4a,b), provide snapshots of the orbitals at the recombination instant $\tau \approx 1.5$ fs after tunnel-ionization, i.e. they contain the relative phase $\Delta \phi_{\mathrm{c}}$ accumulated during the EWP excursion. These images are averaged over $\Delta \tau \approx 600$ as, as given by the electron excursion times spanned by the harmonics (cf. Fig. 3a). The coherent superposition of $\psi_{\text {exp }}^{\text {НОмО }}$ and $\psi_{\text {exp }}^{\text {НОмО-1 }}$ provides time-resolved experimental images of the wavepacket left empty after coherent tunnel ionization from both orbitals ${ }^{16,17}$. Figure 5a shows $\psi_{\text {exp }}^{\text {Hомо }}+$ $\psi_{\text {exp }}^{\text {Ном-1 }}$, i.e. the "hole" at the recombination time. The same wave-packet taken at the tunnel-ionization instant $(\tau=0)$, reconstructed by removing from the HOMO-1 the phase difference $\Delta \phi_{c}=-\pi$ acquired until recombination, i.e. as $\psi_{\exp }^{\text {НОм }}-\psi_{\exp }^{\text {НОо- }}$, is shown in Fig. $5 \mathrm{~b}$. Since the value of $\tau$ coincides approximately with one half-period $\pi /|\Delta \varepsilon|$ of the HOMO-HOMO-1 beating, we observe the motion of the 'hole' from one side of the molecule to the other.

\section{Intra-molecular time-resolved imaging}

Varying the generation parameters (laser intensity and wavelength, selection of the long trajectories) provides different ways for controlling the EWP recombination time, and thus makes it possible to probe the hole at different instants. Combining various phase measurement techniques ${ }^{5,16,23,27}$ (RABITT, 2-source interferometry, gas mixing) with the driving-field polarization control ${ }^{43,44}$ will allow lifting the assumptions made in the tomographic reconstructions, in particular a priori knowledge of the orbital symmetry. This imaging of a dynamic hole wave-packet serves as a test bench for intra-molecular time-resolved imaging. This is a general technique that can be applied to follow the "frontier" orbital of a system during, e.g., a photo-excited process in a conventional pumpprobe scheme. Our progress towards time-resolved tomography thus opens the perspective of imaging ultrafast dynamics of valence electrons and light nuclei at various stages of chemical reactions. 


\section{Methods}

We use the 'Laser UltraCourt Accordable' (LUCA) of the 'Saclay Laser Matter Interaction Center' (SLIC), delivering pulses centered around $795 \mathrm{~nm}$ with up to $30 \mathrm{~mJ}$ energy and $\approx 55 \mathrm{fs}$ duration at a repetition rate of $20 \mathrm{~Hz}$.

Our setup is based on a Mach-Zehnder type interferometer for the `Reconstruction of Attosecond Beating by Interference of Two-photon Transitions' (RABITT) measurement (see Supplementary Information). Drilled mirrors ( $8 \mathrm{~mm}$ hole diameter) separate the annular generating beam (outer diameter cut to $17 \mathrm{~mm}$ by an iris), which contains most of the energy $(\approx 1 \mathrm{~mJ})$, and the weak central part $(\approx 50 \mu \mathrm{J}$, diameter $\approx 4 \mathrm{~mm})$. The latter can be delayed by a piezoeletric translation stage with interferometric stability $(\sim 10 \mathrm{~nm})$. In both arms, combinations of half-wave plate and polarizer allow to finely control the pulse energy. The two beams are then collinearly focused by the same lens of $1 \mathrm{~m}$ focal length $\left(\mathrm{F}_{\#} \approx 60\right.$ for the generating beam and $\mathrm{F}_{\#} \approx 250$ for the probe beam). The beam focus is placed $\approx 5 \mathrm{~mm}$ before the HHG gas jet, thus selecting the short trajectory contribution ${ }^{50}$. An iris of $\approx 4$ $\mathrm{mm}$ diameter blocks the annular generating beam in the far field. The source point of the XUV emission together with the on-axis probe beam is then imaged by a broad-band gold-coated toroidal mirror into the detection volume of a magnetic bottle electron spectrometer (MBES). This toroidal mirror together with a flat mirror cause two grazing-incidence $\left(11.5^{\circ}\right)$ reflections of the HHG radiation on Au-surfaces, preferentially transmitting the s-polarized component into the electron spectrometer (with a 2:1 contrast). The generating laser is kept s-polarized while the molecular alignment axis is rotated in order to predominantly detect the HHG polarization component parallel to the driving laser.

In the spectrometer interaction volume, the neon target gas, injected by a permanent leak, is photoionized by the high harmonics. With the HHG and detection gas jet running, the pressure is $\sim 10^{-3}-10^{-2}$ mbar in the HHG chamber, $\sim 10^{-5}$ mbar in the toroidal mirror chamber, $\sim 10^{-4}$ mbar in the MBES interaction volume and $\sim 10^{-6}$ mbar in the MBES flight tube.

To align molecules in the HHG gas jet, a third laser pulse with controllable delay, polarization and intensity is needed. To this end, a larger interferometer, based on amplitude beam splitters, is set up around the compact RABITT interferometer. One fifth of the incoming pulse energy is transmitted 
into the arm for the aligning beam and passes a motorized delay stage. Here, the stability requirements are much less severe than for the RABITT interferometer, since the variation of the angular distribution of the molecules varies on a $\sim 10$ fs timescale. The central part $(\approx 4$ mm diameter $)$ of this beam is blocked so as to limit the amount of its energy passing into the MBES and thus prevent abovethreshold ionization. The polarization direction of the aligning beam is set by a motorized half-wave plate, thus controlling directly the alignment angle of the molecular ensemble with respect to the HHG driving laser polarization direction at the half-revival delay. The pulse energy in the aligning beam is limited by a diaphragm (cutting the beam to typically $12 \mathrm{~mm}$ diameter), thereby limiting the aligning beam intensity to $\approx 5 \cdot 10^{13} \mathrm{Wcm}^{-2}$ in the HHG gas jet. This also ensures that the aligning beam focal spot is larger than that of the generating beam.

The HHG gas jet has an orifice of $300 \mu \mathrm{m}$ diameter backed with 3 bar pressure. This leads to an estimated $90 \mathrm{~K}$ rotational temperature of the nitrogen molecules at $\approx 1 \mathrm{~mm}$ distance from the orifice, where the generating beam is focused. This estimate is obtained by Fourier transforming the revival trace (dependence of the total harmonic yield on the aligning beam - generating beam delay), and comparing to the Fourier transform of calculated $\left\langle\cos ^{2} \theta\right\rangle$-traces.

\section{Acknowledgements:}

We thank O. Smirnova, M. Ivanov and Y. Mairesse for fruitful discussions. Financial support from the LASERLAB2 program and from the ANR ATTO-WAVE is acknowledged. Parts of the computations have been performed at the Institut du Développement et des Ressources en Informatique Scientifique IDRIS.

\section{Author Contributions :}

P.S. with B.C. planned the project. W.B., P.B. B.C. and P.S. designed and installed the experiment. S.H. with assistance of W.B. P.B. and P.S. performed the measurements. S.H. and J.C. with assistance from W.B.,T.R., Z.D. and P.S. analyzed the data. J.C., C.G.-T., T.A., A.M. and R.T. performed the calculations. All authors discussed the results and contributed to the final manuscript. 


\section{Competing financial interests :}

The authors declare that they have no competing financial interests.

\section{References:}

1. Uiberacker, M. et al. Attosecond real-time observation of electron tunnelling in atoms. Nature 446, 627-632 (2007).

2. Eckle, P. et al. Attosecond ionization and tunnelling delay time measurements in Helium. Science 322, 1525-1529 (2008).

3. Niikura, H. et al. Probing molecular dynamics with attosecond resolution using correlated wave packet pairs. Nature 421, 826-829 (2003).

4. Mauritsson, J. et al. Coherent Electron Scattering Captured by an Attosecond Quantum Stroboscope. Phys. Rev. Lett. 100, 073003 (2008).

5. Mairesse, Y. et al. Attosecond synchronization of high harmonic soft X-ray. Science 302, 1540$1543(2003)$.

6. Sola, I. et al. Controlling attosecond electron dynamics by phase-stabilized polarization gating. Nature Phys. 2, 319-322 (2006).

7. Dudovich, N. L. et al. Measuring and controlling the birth of attosecond XUV pulses. Nature Phys. 2, 781-786 (2006).

8. Cavallieri, A. et al. Attosecond spectroscopy in condensed matter. Nature 449, 1029-1032 (2007).

9. Drescher, M. et al. Time-resolved atomic inner-shell spectroscopy. Nature 419, 803-807 (2002).

10. Fohlisch, A. et al. Direct observation of electron dynamics in the attosecond domain. Nature 436, 373-376 (2005).

11. Haessler et al. Phase-resolved attosecond near-threshold photoionization of molecular nitrogen. Phys. Rev. A 80, 011404 (2009).

12. H. Nakamura. Nonadiabatic Transitions. Concepts, Basic Theories and Applications. (World Scientific Publishing, Singapore, 2002).

13. Breidbach, J. \& Cederbaum, L. S. Universal Attosecond Response to the Removal of an Electron. Phys. Rev. Lett. 94, 033901 (2005). 
14. Hu, S. X. \& Collins, L. A. Attosecond Pump Probe: Exploring Ultrafast Electron Motion inside an Atom. Phys. Rev. Lett. 96, 073004 (2006).

15. Remacle, F. \& Levine, R. D. An electronic time scale in chemistry. PNAS 103, 6793-6798 (2006).

16. Smirnova, O. et al. High harmonic interferometry of multi-electron dynamics in molecules. Nature 460, 972-977 (2009).

17. Smirnova, O. Patchkovskii, S., Mairesse, Y., Dudovich, N. \& Ivanov, M. Yu Strong-field control and spectroscopy of attosecond electron-hole dynamics in molecules. PNAS USA 106, 16556-16561 (2009).

18. Schafer, K. J., Yang, B., DiMauro, L. F. \& Kulander K. C. Above threshold ionization beyond the high harmonic cutoff. Phys. Rev. Lett. 70, 1599-1602 (1993).

19. Corkum, P. B. Plasma perspective on strong field multiphoton ionization. Phys. Rev. Lett. 71, 1994-1997 (1993).

20. Krausz, F. \& Ivanov M. Yu. Attosecond physics. Rev. Mod. Phys. 81, 163-245 (2009).

21. Baker S. et al. Dynamic two-center interference in high-order harmonic generation from molecules with attosecond nuclear motion. Phys. Rev. Lett. 101, 053901 (2008).

22. Li, W. et al., Time-resolved dynamics in $\mathrm{N}_{2} \mathrm{O}_{4}$ probed using high harmonic generation. Science 322, 1207-1211 (2008).

23. Boutu, W. et al. Coherent control of attosecond emission from aligned molecules. Nature Phys. 4, 545-549 (2008).

24. Lein, M. Molecular imaging using recolliding electrons. J. Phys. B 40, R135-R173 (2007).

25. Kanai, T., Minemoto, S. \& Sakai, H. Quantum interference during high-order harmonic generation from aligned molecules. Nature 435, 470474 (2005).

26. Vozzi, C. et al. Controlling two-center interference in molecular high harmonic generation. Phys. Rev. Lett. 95, 153902 (2005).

27. Wagner, N. et al. Extracting the phase of high-order harmonic emission from a molecule using transient alignment in mixed samples. Phys. Rev. A 73, 061403 (2007).

28. Itatani, J. et al. Tomographic imaging of molecular orbitals. Nature 432, 867-871 (2004). 
29. Schwarz, W. H. E. Measuring Orbitals: Provocation or Reality? Angewandte Chemie 45, 15081517 (2006).

30. Walters, Z. B., Tonzani, S. \& Greene, C. H. Limits of the plane wave approximation in the measurement of molecular properties. J. Phys. Chem. 112 9439-9447 (2008).

31. van der Zwan, E., Chirila, C. C. \& Lein, M. Molecular orbital tomography using short laser pulses. Phys. Rev. A 78, 033410 (2008).

32. Le, V.-H., Le, A.-T., Xie, R.-H. \& Lin, C. D. Theoretical analysis of dynamic chemical imaging with lasers using high-order harmonic generation. Phys. Rev. A 76013413 (2007).

33. Torres, R. \& Marangos, J. P. Mapping of orbital structure from high harmonic generation through the molecular dipole moment. J. Mod. Opt. 54, 1883-1899 (2007).

34. Gibson, G. N. \& Biegert, J. Influence of orbital symmetry on high-order-harmonic generation and quantum tomography. Phys. Rev. A 78, 033423 (2008).

35. McFarland, B. K., Farrell, J. P., Bucksbaum, P. H. \& Gühr, M. High harmonic generation from multiple orbitals in $\mathrm{N}_{2}$. Science 322, 1232-1235 (2008).

3-. Lewenstein, M., Balcou, Ph., Ivanov, M. Yu, L'Huillier, A. \& Corkum, P. B. Theory of high harmonic generation by low-frequency laser fields. Phys. Rev. A 49, 2117-2132 (1994).

37. Bransden, B. H. \& Joachain, C. J. Physics of Atoms and Molecules (Pearson Education, Harlow, 2003).

38. Lofthus, A. \& Krupenie, P.H. Spectrum of molecular nitrogen. J. Phys. Chem. Ref. Data 6, 113307 (1977).

39. Tong, X. M., Zhao, Z. X. \& Lin, C. D. Theory of molecular tunneling ionization. Phys. Rev. A 66, $033402(2002)$.

40. Pavicic, D., Lee, K., Rayner, D. M., Corkum, P. B. \& Villeneuve, D. M. Direct Measurement of the Angular Dependence of Ionization for $\mathrm{N}_{2}, \mathrm{O}_{2}$, and $\mathrm{CO}_{2}$ in Intense Laser Fields. Phys. Rev. Lett. 98, 243001 (2007).

41. Kanai, T., Takahashi, E. J., Nabekawa, Y. \& Midorikawa, K. Destructive interference during high harmonic generation in mixed gases. Phys. Rev. Lett. 98, 153904 (2007). 
42. Le, A.T., Lucchese, R. R., Tonzani, S., Morishita, T. \& Lin C. D. Quantitative rescattering theory for high-order harmonic generation from molecules. Phys. Rev. A 80, 013401 (2009).

43. Shafir, D., Mairesse, Y., Villeneuve, D. M., Corkum, P. B. \& Dudovitch, N. Atomic wavefunctions probed through strong-field light-matter interaction. Nature Phys. 5, 412-416 (2009).

44. Mairesse, Y. et al. Electron wavepacket control with elliptically polarized laser light in high harmonic generation from aligned molecules. New J. Phys. 10, 025015 (2008).

45. Schmidt, M. W. et al. General atomic and molecular electronic-structure system. J. Comput. Chem. 14, 1347- 1363 (1993).

46. Smirnova, O. A., Moritzen, S., Patchkovskii, S. \& Ivanov, M. Yu. Coulomb-laser coupling in laser-assisted photoionization and molecular tomography J. Phys. B 40, F197-F206 (2007).

47. Jordan, G. \& Scrinzi, A. Core-polarization effects in molecular high harmonic generation. New J. Phys. 10, 025035 (2008).

48. Patchkovskii, S., Zhao, Z., Brabec, T. \& Villeneuve, D. M. High Harmonic Generation and Molecular Orbital Tomography in Multielectron Systems: Beyond the Single Active Electron Approximation. Phys. Rev. Lett. 97, 123003 (2006).

49. Santra, R. \& Gordon, A. Three-Step Model for High-Harmonic Generation in Many-Electron Systems. Phys. Rev. Lett. 96, 073906 (2006).

50. Balcou, P., Salières, P., L'Huillier, A. \& Lewenstein, M. Generalized phase-matching conditions for high harmonics: The role of field-gradient forces. Phys. Rev. A 55, 3204-3210 (1997). 


\section{Figure Captions :}

Figure 1. Attosecond emission of aligned $\mathbf{N}_{2}$ molecules. (a): Temporal intensity profile of an average pulse in the attosecond pulse train emitted by $\mathrm{N}_{2}$ molecules aligned at angles $\theta=0^{\circ}$ to $90^{\circ}$, reconstructed from a series of RABITT measurements, taking into account harmonics 17 to 27 . Line colours correspond to alignment angles as indicated in Fig. 2. Relative timing calibration was ensured by setting the group delay for the lowest harmonic (H17) to the same value for all angles (see Supplementary Information). The observed evolution thus results from the phase variations within the considered spectral range. While close to our experimental sensitivity limit ( \pm 20 as for the pulse peak positions, see Supplementary Information), the 50-as shift is reliable in view of its regular, almost monotonic (except for the fluctuation at $\theta=60^{\circ}$ ) evolution in the 10 independent measurements. (b, c) Simulated intensity profiles resulting from a coherent superposition of two contributions whose relative weight $\left(\left|a_{2}\right|:\left|a_{1}\right|\right)$ is varied from $0: 1$ to $2: 1$ for all harmonics 17 to 27 . The continuum spectral phases are computed as in Fig. 3a. For the spectral amplitudes (not a sensitive parameter), we used an experimental spectrum $\left(\mathrm{N}_{2}\right.$ at $\left.\theta=0^{\circ}\right)$ for all curves. In (b), $\Delta \phi_{\mathrm{r}}=\pi / 2$ is taken into account by correspondingly shifting the spectral phase for the HOMO contribution, whereas this additional phase is omitted in (c). The $\approx 150$ as overall time-shift between the measured (a) and simulated (b) pulse profiles is presumably due to macroscopic effects (dispersion during propagation), not accounted for in our simulations.

Figure 2. Experimental recombination dipole for $\mathbf{N}_{2}$ molecules. (a): Amplitude and (b) phase of the complex XUV field for $\mathrm{N}_{2}$ at various alignment angles normalized by that for argon. For perpendicular alignment $\left(\theta=90^{\circ}\right)$, the phase decreases by $\pi / 2$ between harmonic 17 and 27 . This jump gradually disappears when rotating the molecules towards parallel alignment $\left(\theta=0^{\circ}\right)$. For all angles, the phase increases by $\pi / 2$ from harmonic 27 on, which might be the beginning of a larger jump not completely contained in our spectral range. The phase difference is set to 0 at the lowest harmonic order 17; see Supplementary Information for details on the data analysis. 
Figure 3. Phase of the harmonic emission. (a): Continuum phase difference $\Delta \phi_{c}\left(q \omega_{\mathrm{L}}\right)$ computed within SFA for an $800 \mathrm{~nm}$ laser field with intensity $1.2 \cdot 10^{14} \mathrm{Wcm}^{-2}$ and binding energies $\varepsilon_{1}=-15.58 \mathrm{eV}$ and $\varepsilon_{2}=-17.00 \mathrm{eV}$, corresponding to the $\mathrm{HOMO}$ and HOMO-1 contributions (short trajectories are considered). Horizontal lines indicate the phase differences and excursion times for the first (H17), middle (H25) and last (H31) harmonics of the experimental range (grey area). (b, c): Phases of the HOMO recombination dipole computed with Coulomb-waves, at 3 different orientations $\theta$, vs. electron momentum $k$. For computational purposes, the orbital was expressed as a combination of single-center Slater functions. Two values of the effective charge are considered for the Coulomb waves: (b) $Z^{*}=0$, corresponding to plane waves, and (c) $Z^{*}=1$, corresponding to the asymptotic charge of $\mathrm{N}_{2}^{+}$. For $\mathrm{k}>0.5$ a.u., both series of curves show similar patterns, up to a $k$-dependent global phase shift and a translation $\delta k \approx 0.4$ a.u. on the momentum scale. For $k<0.5$ a.u., the fast oscillations in (c) are a direct imprint left by the Coulomb-waves, as can be seen from their partial wave-expansion ${ }^{37}$, where each angular momentum $l$ contributes with a phase $\eta_{l}\left(Z^{*}, k\right)=\arg \left[\Gamma\left(l+1+\mathrm{i} Z^{*} / k\right)\right]$.

Figure 4. Orbital tomographic reconstructions. (a): from the imaginary part of the experimental dipole imposing $\sigma_{\mathrm{g}}$-symmetry; (b) : from the real part of the experimental dipole imposing $\pi_{\mathrm{u}^{-}}$ symmetry; (c) : simulated reconstruction with the HOMO PW-dipole; (d) : simulated reconstruction with the HOMO-1 PW-dipole. For the sake of comparison, simulations were done with the experimental sampling (harmonics and angles, see Fig. 2), using the dispersion relation $k_{q}{ }^{2} / 2=q \omega_{\mathrm{L}}$, and by restricting the theoretical dipole to its component along the laser polarization. Black dots indicate the nuclei position at equilibrium distance. Reconstructions in $(\mathbf{b}, \mathbf{d})$, are based on the 'velocity-form' of the dipole to avoid division by $\mathrm{x} / \mathrm{y}$ in real space and thus numerical problems with the orbital nodes at $\mathrm{y}=0$. (e): Hartree-Fock HOMO and (f): HOMO-1, calculated with GAMESS ${ }^{45}$. 
Figure 5. Reconstructions of the dynamic hole. (a): Squared sum and (b): squared difference of the wavefunctions shown in Figure 4a,b. These can be interpreted as the hole-density in the ion at the recollision / tunnel-ionization instants (see text) for a relative weight of the HOMO:HOMO-1 contributions corresponding to the relative amplitudes of the wavefunctions in Figure 4a,b. Although we have access to wave-packets, we plot the corresponding densities so as to visually emphasize their asymmetries. 

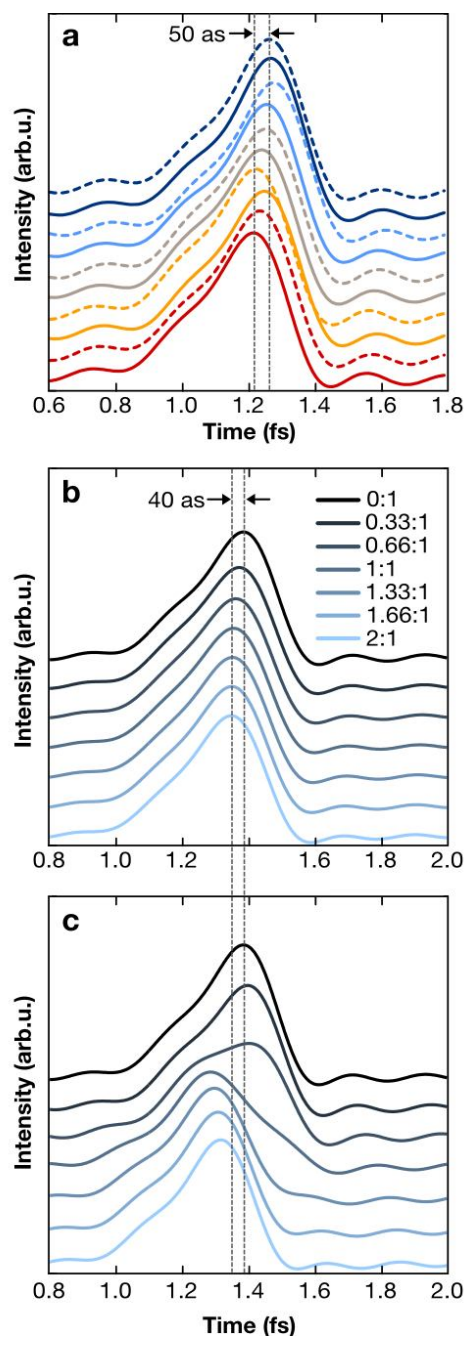

Fig.1
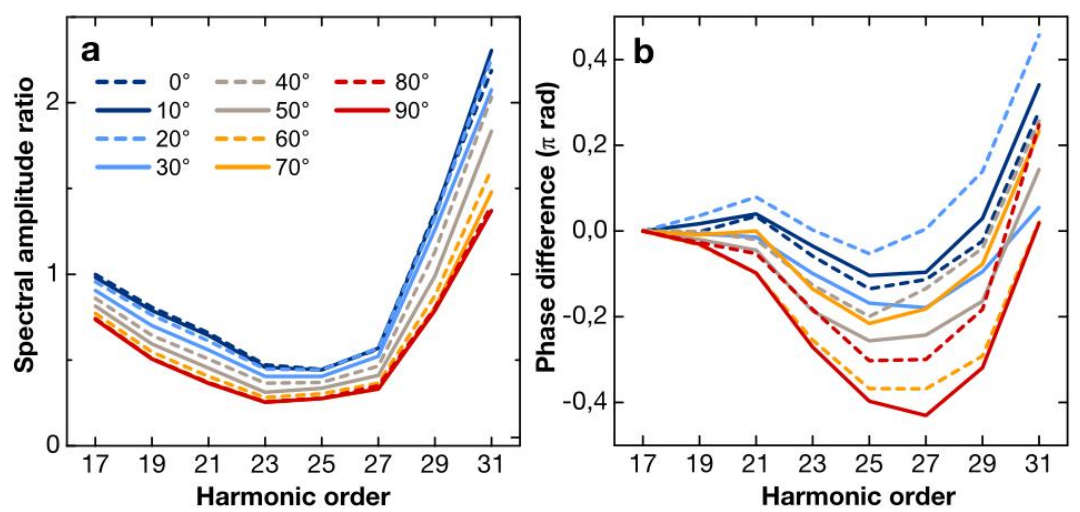

Fig.2 

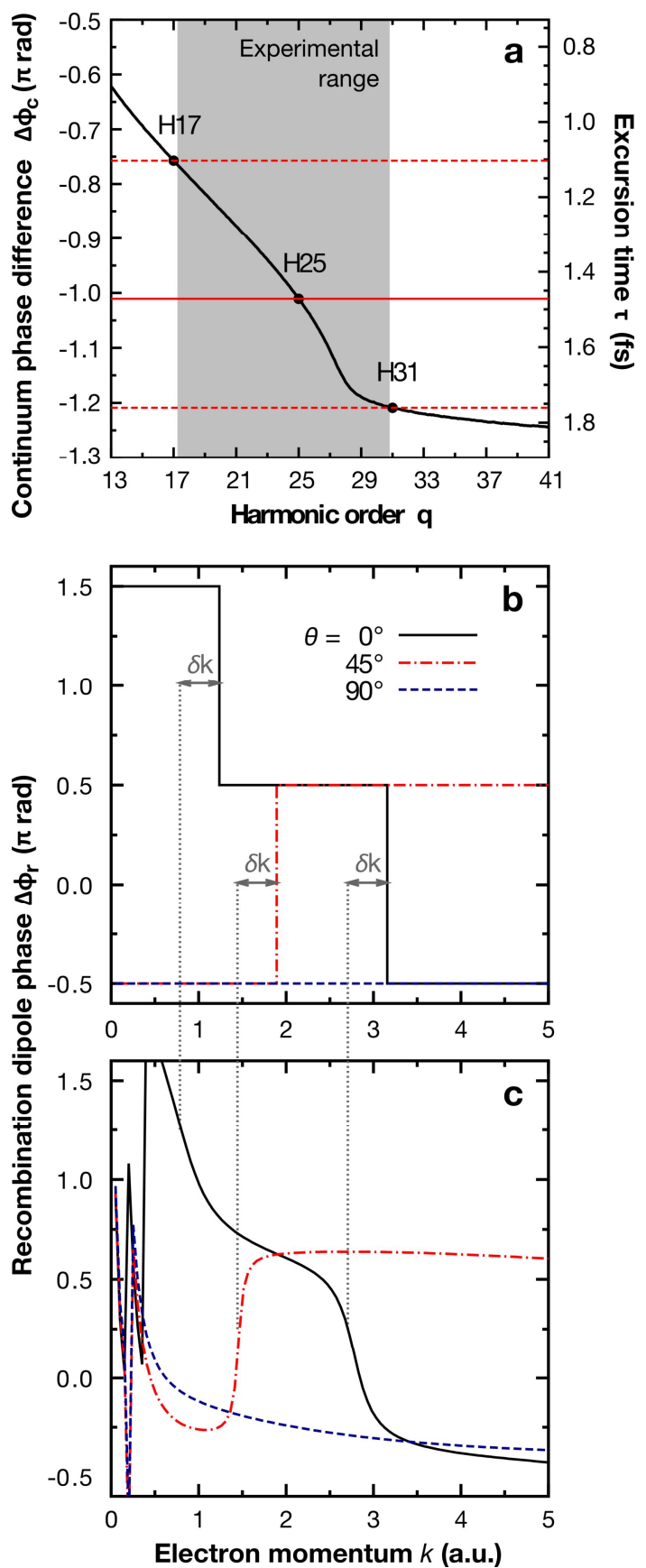

Fig. 3 


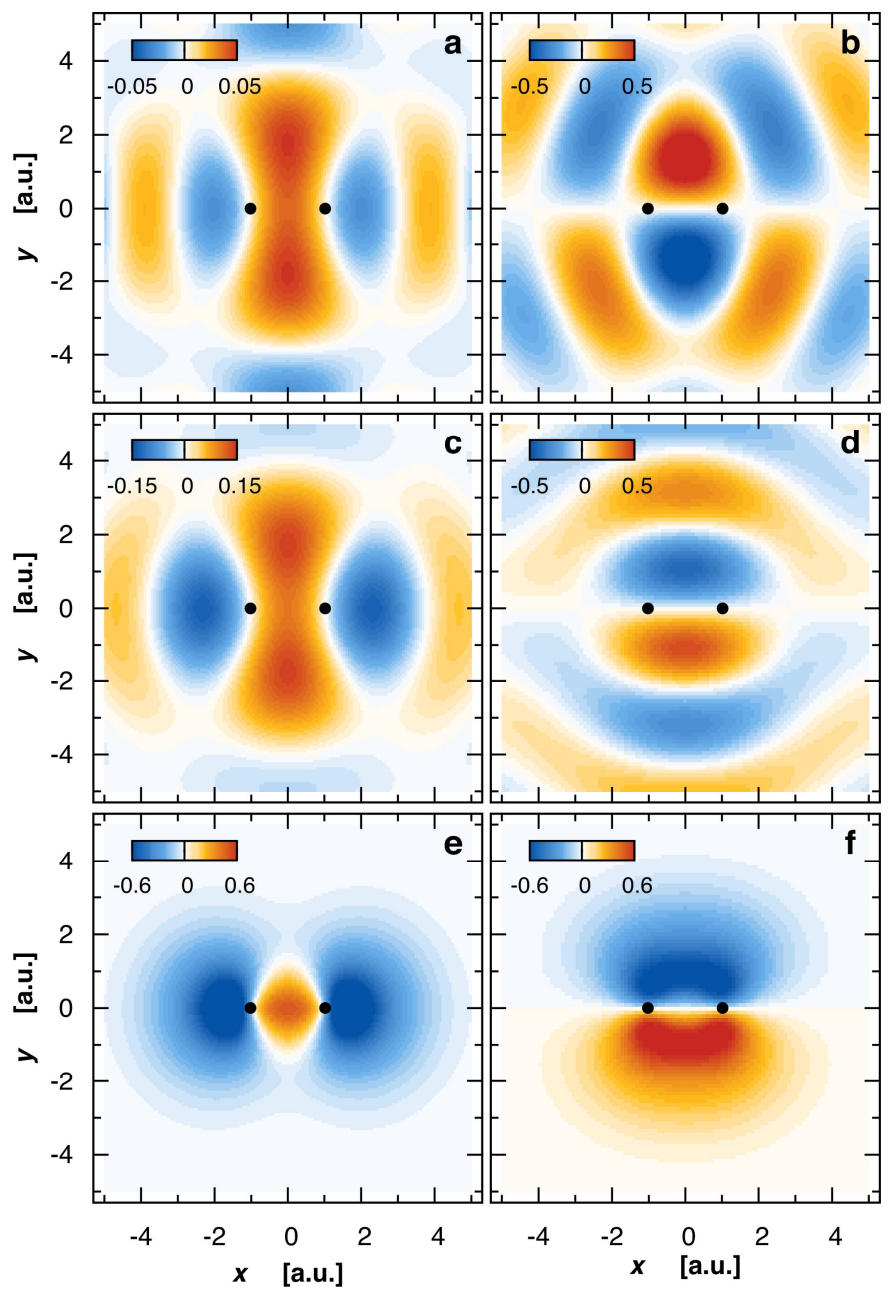

Fig. 4

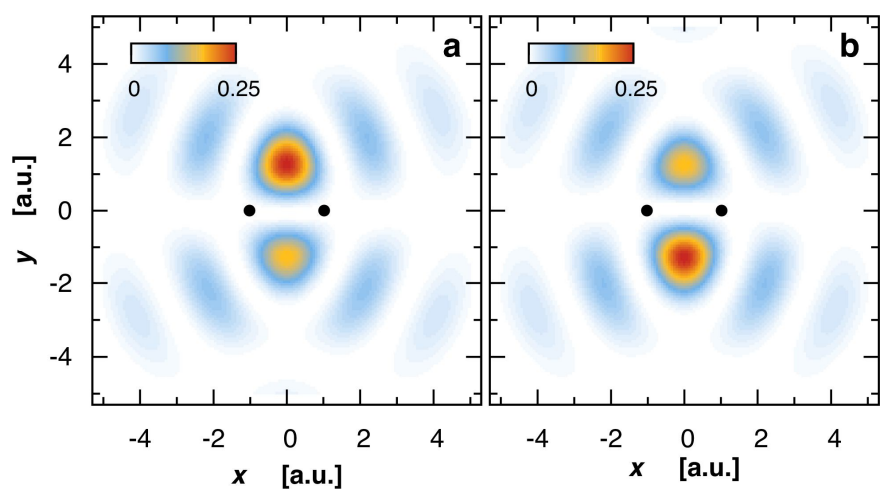

Fig. 5 